\title{
Evidence against an association between gamma-ray bursts and Type I supernovae
}

\author{
C. Graziani, D.Q. Lamb, and G.H. Marion
}

Department of Astronomy and Astrophysics, University of Chicago, 5640 South Ellis Avenue, Chicago, IL 60637, U.S.A. Received December 29, 1998; accepted April 29, 1999

\begin{abstract}
We use a rigorous method, based on Bayesian inference, for calculating the odds favoring the hypothesis that any particular class of astronomical transients produce gamma-ray bursts over the hypothesis that they do not. We then apply this method to a sample of 83 Type Ia supernovae and a sample of 20 Type Ib-Ic supernovae. We find overwhelming odds against the hypothesis that all Type Ia supernovae produce gamma-ray bursts, whether at low redshift $\left(10^{9}: 1\right)$ or high-redshift $\left(10^{12}: 1\right)$, and very large odds (6000:1) against the hypothesis that all Type $\mathrm{Ib}, \mathrm{Ib} / \mathrm{c}$, and Ic supernovae produce observable gamma-ray bursts. We find large odds (34:1) against the hypothesis that a fraction of Type Ia supernovae produce observable gamma-ray bursts, and moderate odds (6:1) against the hypothesis that a fraction of Type Ib-Ic supernovae produce observable bursts.
\end{abstract}

Key words: gamma-rays: bursts — stars: supernovae, general

\section{Introduction}

The discovery of supernova 1998bw (Galama et al. 1998) within the 8 arcmin radius of the BepppoSAX WFC error circle for GRB 980425 (Soffitta et al. 1998) has led to the hypothesis that some GRB sources are Type Ib-Ic SNe.

There are some serious difficulties with this interpretation of the data for GRB 980425/SN 1998bw: the supernova occurred outside the NFI error circle of a fading X-ray source (Pian et al. 1998a, 1998b, 1999; Piro et al. 1998). This source had a temporal decay consistent with a power-law index of $\sim 1.2$ (Pian et al. 1998b), which resembles the temporal behavior of X-ray afterglows seen in almost every other GRB followed up with the SAX NFI. It must therefore be viewed as a strong candidate to be the X-ray afterglow of GRB 980425.

Moreover, if the association between GRB 980425 and SN 1998bw were true, the luminosity of this burst would be $\sim 10^{46} \mathrm{erg} \mathrm{s}^{-1}$ and its energy would be $\sim 10^{47} \mathrm{erg}$. Each would therefore be five orders of magnitude less than that of other bursts, and the behavior of the X-ray and optical afterglow would be very different from those of the other BeppoSAX bursts, yet the burst itself is indistinguishable from other BeppoSAX and BATSE GRBs with respect to duration, time history, spectral shape, peak flux, and and fluence (Galama et al. 1998).
In view of these difficulties, the safest procedure is to regard the association as a hypothesis that is to be tested by searching for correlations between SNe and GRB in catalogs of SNe and GRBs, excluding SN 1998bw and GRB 980425. Wang \& Wheeler (1998a; see also Wang \& Wheeler 1998b) have correlated BATSE GRB with Type Ia and with Type Ib-Ic Sne. They found that the data was "consistent" with the assumption of an association between GRB and Type Ib-Ic SNe.

In the present work we improve upon the Wang \& Wheeler correlative study by introducing an analysis method based on Bayesian inference, and therefore using the likelihood function, that incorporates information about the BATSE position errors in a non-arbitrary way and that is free of the ambiguities of a posteriori statistics. The method also accounts for the fact that the BATSE temporal exposure is less than unity.

\section{Methodology}

We use the BATSE 4B GRB Catalog (Meegan et al. 1998), and BATSE bursts that occurred subsequent to the $4 \mathrm{~B}$ catalog but before 1 May 1998. We also use the Ulysses supplement to the BATSE 4B catalog, which contains 219 BATSE bursts for which 3rd IPN annuli have been determined (Hurley et al. 1998). Hurley (private communication, 1998) has kindly made available at our request 3rd IPN annuli for an additional 9 BATSE bursts that occurred subsequent to the period of the BATSE $4 \mathrm{~B}$ catalog but before 1 May 1998.

We have compiled three Type I SNe samples. The first is a sample of 37 Type Ia SNe at low redshift $(z<0.1)$ from the CfA SN Search Team (Riess 1998, private communication). The second is a sample of 46 moderate redshift $(0.1<z<0.830)$ Type Ia SNe from the Supernova Cosmology Project (Perlmutter 1998, private communication). The third sample consists of 20 Type Ib, Ib/c, and Ic SNe compiled from IAU Circulars and various $\mathrm{SNe}$ catalogs.

We compare three hypotheses: $H_{1}$ : The association between SNe and GRBs is real. If a SN is observed, there is a chance $\epsilon$ that BATSE sees the associated GRB, where $\epsilon$ is the average BATSE temporal exposure. While $\epsilon$ varies with Declination, the variation is modest and we neglect it. The probability density for the time of occurrence of the $i$ th supernova is assumed uniform in the interval $\left[t_{i}, t_{i}+\tau_{i}\right]$, 
so that all GRBs that occur in that interval have an equal prior probability of being associated with the SN. $H_{1}^{\prime}$ : The association is real, but only a fraction $f$ of detectable SNe produce detectable GRB. $\mathrm{H}_{2}$ : There is no association between $\mathrm{SNe}$ and GRBs.

We calculate the Bayesian Odds, $\mathcal{O}$ favoring $H_{1}$ over $\mathrm{H}_{2}$. The details of the calculation are presented in (Graziani et al. 1998). We separately compare $H_{1}^{\prime}$ to $H_{2}$, denoting the odds favoring $H_{1}^{\prime}$ over $H_{2}$ by $\mathcal{O}^{\prime}$. Finally, we calculate the posterior probability distribution for the parameter $f$ in the model $H_{1}^{\prime}$, and infer an upper limit on its value.

\section{Results}

We find overwhelming odds against the hypothesis that all Type Ia supernovae produce gamma-ray bursts, whether at low redshift $\left(\mathcal{O}=10^{9}: 1\right)$ or high-redshift $\left(\mathcal{O}=10^{12}: 1\right)$. We also find large odds $\left(\mathcal{O}^{\prime}=34: 1\right)$ against the hypothesis that a fraction of Type Ia supernovae produce observable gamma-ray bursts.

We find very large odds $(\mathcal{O}=6000: 1)$ against the hypothesis that all Type Ib, Ib/c, and Ic supernovae produce observable gamma-ray bursts. We also find moderate odds $\left(\mathcal{O}^{\prime}=6: 1\right)$ against the hypothesis that a fraction of Type Ib-Ic supernovae produce observable bursts. If we nevertheless assume that this hypothesis is correct, we find that the fraction $f_{\mathrm{SN}}$ of Type Ib, Ib/c and Ic SNe that produce observable GRBs must be less than $0.17,0.42$, and 0.70 with $68 \%, 95 \%$, and $99.6 \%$ probability, respectively. These limits are relatively weak because of the modest size (20 events) of our sample of Type Ib-Ic SNe.

\section{Discussion}

We find large odds against the hypothesis that all Type Ib, Ib/c, and Ic supernovae produce observable gamma-ray bursts, the specific hypothesis considered by Wang \& Wheeler (1998a, 1998b). The odds against the hypothesis that a fraction of Type Ib-Ic supernovae produce observable bursts are more moderate, because they account for the possibility that $f$ is so small that no SN-produced GRBs are detected, in which case the data can not distinguish between $H_{1}^{\prime}$ and $H_{2}$.

Type Ib, Ib/c and Ic SNe are now being found at a rate of about eight a year, so that the size of the sample of known Type Ib-Ic SNe should triple within about five years. One might hope that future analyses, using the statistical methodology that we have presented here, could either show that the association between Type Ib-Ic SNe and GRBs is rare, or confirm the proposed association. Unfortunately, achieving the former will be difficult: the limit on the fraction $f_{\mathrm{SN}}$ of Type Ib-Ic SNe that produce observable GRBs scales like $N_{\mathrm{SN}}^{-1}$ for large $N_{\mathrm{SN}}$, and therefore tripling the size of the sample of known Type Ib-Ic SNe without observing an additional possible SN - GRB association would only reduce the $99.7 \%$ probability upper limit on $f_{\mathrm{SN}}$ to 0.24 .

The parameter $f$ represents the fraction of observable SNe which can produce observable GRBs. Some observ- able SNe might not produce observable GRBs because of intrinsic effects, such as beaming, whereas others might not do so because the sampling distance for $\mathrm{SN}$-produced GRB could be less than the sampling distance for the $\mathrm{SNe}$ themselves. It is possible to separate these two effects, by writing $f=f_{\text {intrinsic }} \times f_{\text {sampling. }}$. Obviously, the more interesting quantity is $f_{\text {intrinsic }}$, since it addresses the nature of the GRB sources.

We may attempt to find a constraint on $f_{\text {intrinsic }}$ by assuming the correctness of the identification of SN 1998bw with GRB 980425 and using that association to estimate $f_{\text {sampling, }}$, under the (dubious) assumption that the GRBs produced by Type Ib-Ic SNe are standard candles. The result is $f_{\text {sampling }} \approx 1.910^{-3}$. This is rather bad news for the prospect of constraining $f_{\text {intrinsic }}$, since the product of $f_{\text {intrinsic }}$ and $f_{\text {sampling }}$ is only constrained by the data to be less than about 0.7 . Thus this argument can place no constraint on $f_{\text {intrinsic }}$. Elsewhere, we show that placing a meaningful constraint on $f_{\text {intrinsic }}$ would require a GRB experiment approximately 80 times more sensitive than BATSE (Graziani et al. 1998).

One can also approach the proposed association between SNe and GRBs from the opposite direction. The interesting question, from this point of view, is what fraction $f_{\mathrm{GRB}}$ of the GRBs detected by BATSE could have been produced by Type Ib-Ic SNe? (note that this is different from the question addressed by Kippen et al. 1998, who constrained the fraction of BATSE GRB that could have been produced by known $\mathrm{SNe}$ ). Such a limit may be derived under the same assumptions as made in the previous paragraph. We find that no more than $\sim 90$ SNe could have produced GRB detectable by BATSE, indicating that $f_{\mathrm{GRB}}$ can be no more than about $5 \%$.

\section{References}

Galama T.J., et al., 1998, Nat (submitted) (astro-ph/9806175) Graziani C., Lamb D.Q., Marion G.H., 1998, ApJ (submitted) (astro-ph/9810374)

Hakkila J., Meegan C.A., Pendleton G.N., Henze W., McCollough M., Kommers J.M., Briggs M.S., 1998, in Gamma-Ray Bursts, AIP Conf. Proc. 428, Meegan C.A., Preece R.D., Kogut T.M. (eds.). New York: AIP, p. 509

Hurley K., et al., 1998, ApJS (submitted) (data are publicly available at http://ssl.berkeleyedu/ipn3.index.html)

Kippen R.M., et al., 1998, ApJL 506, L27

Meegan C.A., et al., 1998, in Gamma-Ray Bursts, AIP Conference Proceedings 428, Meegan C.A., Preece R.D., Kogut T.M. (eds.). New York: AIP, p. 3

Pian E., Antonelli L.A., Daniele M.R., et al., 1998, GCN Report 61

Pian E., Frontera F., Antonelli L.A., Piro L., 1998, GCN Report 69

Pian E., et al., 1999 (these proceedings)

Piro L., Butler R.C., Fiore F., Antonelli A., Pian E., 1998, GCN Report 155

Soffitta P., et al., 1998, IAU Circ. 6884

Wang L., Wheeler J.C., 1998a, astro-ph/9806212

Wang L., Wheeler J.C., 1998b, ApJ 504, L87 